\title{
Antagonismo na Associação de Glyphosate e Triazinas ${ }^{1}$
}

\author{
Antagonism with Glyphosate and Triazine Combinations \\ VIDAL, R.A. ${ }^{2}$, MACHRY, M. ${ }^{3}$, HERNANDES, G.C. ${ }^{4}$ e FLECK, N.G. ${ }^{2}$
}

\begin{abstract}
RESUMO - No sistema de semeadura direta, há a necessidade de realizar o controle da vegetação existente na área antes da semeadura da cultura e também de utilizar herbicidas residuais para evitar a infestação de plantas daninhas no período inicial do desenvolvimento da cultura. É interesse dos agricultores utilizar herbicidas dessecantes e residuais simultaneamente, a fim de economizar tempo e recursos financeiros. O objetivo deste trabalho foi verificar a compatibilidade de glyphosate e triazinas quando associados no tanque do pulverizador. O experimento foi realizado em campo, na safra de crescimento 2001-2002. Utilizou-se como planta reagente o sorgo, híbrido Dekalb 865, semeado em novembro de 2001. Os tratamentos herbicidas foram aplicados 60 dias após a semeadura, quando as plantas estavam com $1,0 \mathrm{~m}$, no início do emborrachamento. Os tratamentos consistiram de glyphosate nas doses de 540, 720 e $900 \mathrm{~g} \mathrm{ha}^{-1}$, aplicado isoladamente ou associado a simazine + atrazine (Primatop SC) nas doses de $1.750+1.750 \mathrm{~g} \mathrm{ha}^{-1}$. Também foram testados um tratamento sem herbicidas e outro apenas com o herbicida residual. As avaliações de controle e de teores de clorofila indicaram efeitos antagônicos para as três doses de glyphosate. Os efeitos antagônicos da associação dos herbicidas reduzem de intensidade com o aumento da dose do glyphosate ou com o decorrer do tempo.
\end{abstract}

Palavras-chave: plantio direto, Sorghum bicolor, mistura, atrazine, simazine.

\begin{abstract}
The no-tillage system requires herbicide spraying before planting to eliminate the existing weeds in the area and residual herbicides to prevent new weed infestation during the crop cycle. Farmers tend to spray both groups of herbicide simultaneously to reduce costs and application time. The objective of this work was to verify the compatibility of glyphosate and triazine as a tank mix. An experiment was conducted under field conditions during the 20012002 growing season, using sorghum as a model to evaluate the herbicide mixture compatibility. Sorghum hibrid Dekalb 865 was planted in November 2001 and the treatments were sprayed 60 days after planting, when the plants were $100 \mathrm{~cm}$ tall and at the boot stage. Treatments consisted of glyphosate at 540, 720 and $900 \mathrm{~g} \mathrm{ha}^{1}$, sprayed alone or in mixture with simazine + atrazine (Primatop SC) at $1750+1750 \mathrm{~g} \mathrm{ha}{ }^{1}$. Treatments also included an untreated control and the residual herbicides used alone. The results of plant control and chlorophyll content indicated a severe antagonism in the glyphosate and triazine combination. Herbicide antagonism reduced with increment of glyphosate rate or at late assessment periods.
\end{abstract}

Key words: no-tillage, Sorghum bicolor, mixture, atrazine, simazine.

\section{INTRODUÇÃO}

O sistema de semeadura direta (SPD), popularmente denominado plantio direto, foi introduzido no início da década de 70, com o objetivo principal de controlar a erosão (Borges, 1993). Além das alterações no preparo do solo, o SPD modificou também a forma de controle

1 Recebido para publicação em 2.1.2003 e na forma revisada em 11.8.2003.

2 Eng.-Agr., Ph.D., Prof. da Faculdade de Agronomia da Universidade Federal do Rio Grande do Sul - UFRGS, Caixa Postal 15.100, 90001-970, Porto Alegre-RS, Bolsista do CNPq, <ribas.vidal@ufrgs.br>; ${ }^{3}$ Eng.-Agr., M.S.; ${ }^{4}$ Aluno de mestrado do Programa de Pós-Graduação em Fitotecnia da UFRGS.

Planta Daninha, Viçosa-MG, v.21, n.2, p.301-306, 2003 
das plantas daninhas. Capinas manuais ou mecânicas, usadas no sistema de preparo convencional do solo, foram substituídas pelo manejo químico através de aplicações de herbicidas. Na cultura de milho, o controle das plantas daninhas no SPD passou a ser realizado com herbicidas residuais aplicados em pré ou pós-semeadura (Vidal \& Spader, 1999).

A introdução, no Brasil, do herbicida glyphosate ocorreu em meados dos anos $70 \mathrm{e}$ possibilitou o controle satisfatório de plantas daninhas já emergidas antes da semeadura da cultura no SPD (Velloso \& Souza, 1993). Herbicidas com este ingrediente ativo perfazem $12 \%$ das vendas globais de herbicidas, somando mais de 150 marcas comerciais (Trezzi et al., 2001). O glyphosate é um herbicida sistêmico, de ação não-seletiva, que inibe a enzima enolpiruvil-shiquimato fosfato sintase (EPSPS) e provoca a morte de plantas anuais e perenes (Kruse et al., 2000).

Atualmente, o controle das plantas daninhas em SPD é realizado com uma aplicação de glyphosate antes da semeadura e uma de herbicidas residuais, seletivos à cultura, em pré-emergência, ou com aplicações de produtos seletivos em pós-emergência da cultura e das plantas daninhas. Nos cultivos de leguminosas, como soja e feijão, o uso de produtos de uso pós-emergente e seletivos tem sido mais freqüente que no cultivo de cereais, como milho e sorgo. No cultivo destes cereais, merece destaque o uso de herbicidas do grupo das triazinas, que inibem o fluxo de elétrons no fotossistema II (FS2), sendo aplicados em pré ou em pósemergência inicial para o controle de plantas daninhas dicotiledôneas e monocotiledôneas (Fleck \& Vidal, 2001).

Como o controle de plantas daninhas no SPD requer dois ou mais tipos de produtos, os produtores têm efetuado associações, popularmente referidas como misturas, de herbicidas no tanque do pulverizador, com a finalidade de reduzir o número de pulverizações. A prática mais comum é aspergir em uma só ocasião herbicidas de ação total e residuais. Nessas associações, destaca-se a mistura dos herbicidas glyphosate e triazinas.

Associações entre dois ou mais herbicidas é prática comum para a maioria das culturas e objetiva aumentar o espectro de espécies controladas (Damalas \& Eleftherohorinos, 2001) ou aumentar o periodo de controle destas (Vangessel et al., 2000). Efeitos de sinergismo podem ser observados em associações de glyphosate e imazethapyr (Starke \& Oliver, 1998). Contudo, muitas associações de glyphosate com outros herbicidas podem resultar em antagonismo entre os produtos.

A possibilidade ou não de ocorrência de antagonismo depende dos herbicidas associados e da planta daninha a ser controlada. Associações de glyphosate com CGA 2774776, chlorimuron, cloransulan-methyl, fomesafen, imazaquin ou pyrithiobac resultaram em antagonismo no controle de Ipomoea lacunosa L. (Shaw \& Arnold, 2002) . Já para Sorghum halepense e Brachiaria plathyphylla essas associações não apresentaram efeitos antagônicos.

Associação de glyphosate com sulfentrazone é antagônica no controle de Amaranthus palmeri, Echinochloa crus-galli, Ipomoea hederaceae e Eleusine indica (Starke \& Oliver, 1998). Efeitos antagônicos em associação de glyphosate com atrazina e alachlor foram observados quando comparados com aplicações isoladas destes produtos para o controle de Agropyron repens (Selleck \& Baird, 1981).

Ocorre variabilidade no antagonismo observado nas associações entre atrazine e glyphosate. $\mathrm{O}$ antagonismo na mistura destes herbicidas depende de formulações de atrazine, levando à conclusão de que os ingredientes inertes influenciam esse processo (Stahlman $\&$ Phillips, 1979). A redução na eficiência do glyphosate em associações com atrazine ocorre em função da mistura destes produtos na calda de pulverização e não por interação biológica na planta (Appleby \& Somabhi, 1978). O antagonismo constatado em ensaios a campo nem sempre se repete em experimentos realizados em casa de vegetação, sugerindo que esses efeitos antagônicos podem estar relacionados com condições ambientais ocorrentes durante a aplicação dos herbicidas no campo (Bradley et al., 2000).

O objetivo deste trabalho foi avaliar o efeito da interação entre os herbicidas glyphosate e triazinas misturados no tanque de pulverização para o controle de plantas de sorgo que se desenvolviam no campo. 


\section{MATERIAL E MÉTODOS}

O experimento foi instalado no ano agrícola de 2001/2002, na Estação Experimental Agronômica da Universidade Federal do Rio Grande do Sul (EEA/UFRGS), localizada em Eldorado do Sul-RS, em solo Argissolo Vermelho Distrófico típico com $380 \mathrm{~g} \mathrm{~kg}^{-1}$ de argila, $24 \mathrm{~g} \mathrm{~kg}^{-1}$ de matéria orgânica, $5 \mathrm{mg} \mathrm{dm}{ }^{-3} \mathrm{de} \mathrm{P}$, $140 \mathrm{mg} \mathrm{dm}^{-3}$ de K e pH de 5,5.

O delineamento experimental foi o de blocos ao acaso, com quatro repetições. Os tratamentos consistiram de glyphosate (Roundup Original) nas doses de 540, 720 e $900 \mathrm{~g} \mathrm{ha}^{-1}$, aplicado sozinho ou associado a simazine + atrazine (Primatop SC) nas doses de $1.750+$ $1.750 \mathrm{~g} \mathrm{ha}^{-1}$. Também foram testados um tratamento sem herbicidas e outro apenas com o herbicida residual. As doses de glyphosate foram selecionadas para testar a hipótese de que triazinas conferem antagonismo ao glyphosate. A dose selecionada para as triazinas foi a máxima recomendada na bula do produto. No tratamento com aplicação isolada de atrazine e simazine, as plantas de sorgo foram cortadas, sendo retirada a palha para a aplicação do herbicida diretamente ao solo, a fim de investigar um possivel efeito residual. A dimensão das parcelas foi de $3 \times 10 \mathrm{~m}$.

A planta-alvo foi o sorgo DKB 865, semeado em 5.11.2001 com densidade de $15 \mathrm{~kg} \mathrm{ha}^{-1}$ de sementes, com espaçamento de $17 \mathrm{~cm}$ entre linhas. Por ocasião do plantio foi efetuada fertilização com $200 \mathrm{~kg} \mathrm{ha}^{-1}$ de adubo da fórmula 5-20-20 (N-P-K). O sorgo, no momento do tratamento, encontrava-se no estádio de nove folhas (100 cm de altura). Os tratamentos foram aplicados no dia 9.1.2002 às 14 horas; a temperatura do ar era de $25^{\circ} \mathrm{C}$ e a umidade relativa do ar de $60 \%$, sem ocorrência de ventos e com nebulosidade de 95\%. Foi utilizado equipamento costal de precisão, pressurizado com $\mathrm{CO}_{2}$, com barra de quatro bicos espaçados de $50 \mathrm{~cm}$, contendo pontas XR 8002, mantendose pressão constante de $200 \mathrm{kPa}$ durante a aplicação. A velocidade de deslocamento foi de $1 \mathrm{~m} \mathrm{~s}^{-1}$, com volume de calda de $220 \mathrm{~L} \mathrm{ha}^{-1}$. Nos tratamentos com associação de herbicidas a preparação da calda foi efetuada 30 minutos antes da aplicação.

O controle das plantas de sorgo foi avaliado visualmente aos 15, 30, 45 e 60 dias após a aplicação dos tratamentos (DAT), de modo comparativo à testemunha não-tratada, utilizando-se escala percentual, na qual a nota zero significou ausência de controle e a nota 100 representou controle total das plantas. Também foi realizada, aos 30 DAT, leitura correspondente ao teor de clorofila na folha, utilizando-se clorofilômetro Minolta SPAD-502, por meio de determinações realizadas na última folha completamente expandida, em 10 plantas por parcela.

Os resultados obtidos foram submetidos à análise de variância e, quando o teste $\mathrm{F}$ mostrou significância a $5 \%$ de probabilidade, as médias foram comparadas pelo teste t. Para comparar os efeitos de doses, realizou-se a análise de regressão para a respectiva variávelresposta, sendo utilizados modelos linear e quadrático para ajustar a distribuição dos dados obtidos.

\section{RESULTADOS E DISCUSSÕES}

As avaliações de controle de plantas daninhas realizadas em todas as épocas não evidenciaram infestações. Assim, apenas os resultados de controle de sorgo foram analisados e discutidos neste trabalho, não sendo considerado na análise o tratamento com herbicidas residuais apenas.

$\mathrm{Na}$ avaliação de controle efetuada aos 15 DAT (Figura 1) foram observados efeitos antagônicos para a associação de glyphosate e triazinas em relação ao glyphosate sozinho, em qualquer das doses de glyphosate testadas. Quando o glyphosate foi aplicado isoladamente, mesmo na menor dose testada, obteve-se bom controle do sorgo, sendo superior à maior dose de glyphosate aplicado em associação com triazinas. O efeito de dose de glyphosate dependeu da associação de herbicidas no tanque. Glyphosate aplicado isoladamente proporcionou resposta quadrática no controle do sorgo. Quando ele foi aplicado em associação com triazinas, obteve-se efeito linear positivo, com aumento de $0,05 \%$ no controle de sorgo para cada g ha-1 de glyphosate utilizado (Figura 1).

$\mathrm{Na}$ avaliação de controle efetuada aos 30 DAT (Figura 2), observa-se também o efeito antagônico para associação de glyphosate com triazinas. O controle do sorgo apresentou respostas lineares positivas, com 0,1 e $0,07 \%$ 
de incremento para cada $\mathrm{g} \mathrm{ha}^{-1}$ de glyphosate utilizado nas aplicações isoladas e em associação com triazinas, respectivamente.

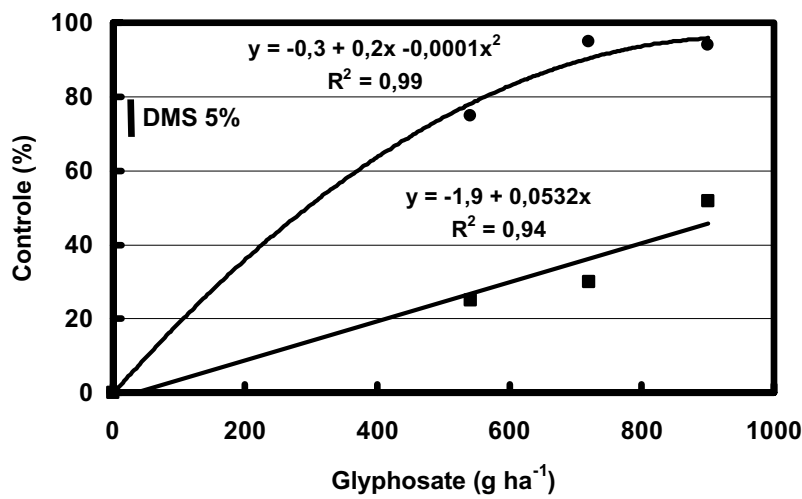

Figura 1 - Controle de sorgo avaliado aos 15 dias após a aplicação de glyphosate isoladamente $(\bullet)$ ou associado a triazinas (ם). Barra vertical para comparar médias de tratamentos entre equações pelo teste $t(\mathrm{P}<0,05)$. Todas as equações foram significativas $(\mathrm{P}<0,05)$. Eldorado do Sul-RS, 2002.

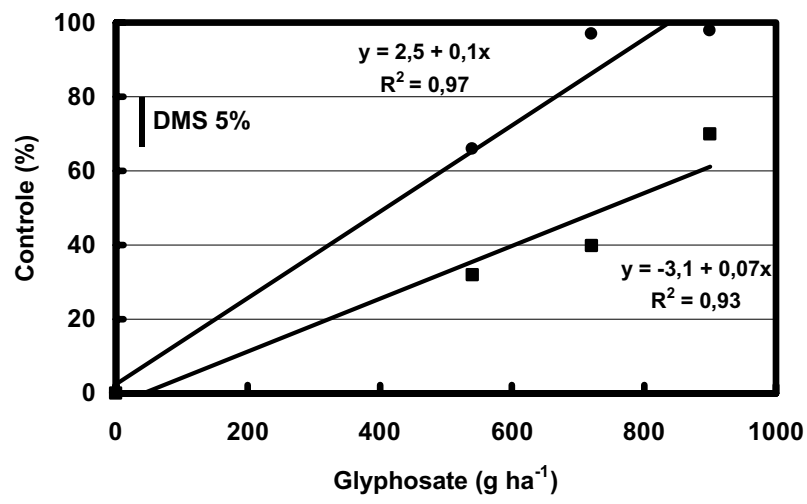

Figura 2 - Controle de sorgo avaliado aos 30 dias após a aplicação de glyphosate isoladamente $(\bullet)$ ou associado a triazinas (ם). Barra vertical para comparar médias de tratamentos entre equações pelo teste $\mathrm{t}(\mathrm{P}<0,05)$. Todas as equações foram significativas $(\mathrm{P}<0,05)$. Eldorado do Sul-RS, 2002

$\mathrm{Na}$ avaliação de controle realizada aos 45 DAT (Figura 3), foram observados efeitos antagônicos entre glyphosate e triazinas somente nas doses de 540 e $720 \mathrm{~g} \mathrm{ha}^{-1}$ de glyphosate. Para a dose de $900 \mathrm{~g} \mathrm{ha}^{-1}$ não foi verificado efeito antagônico. Nesta avaliação, a aplicação isolada de glyphosate proporcionou resposta quadrática positiva para o controle do sorgo com o incremento da dose de glyphosate. Na aplicação dos herbicidas associados, observa-se resposta linear de controle do sorgo, com $0,1 \%$ de incremento no controle por $\mathrm{g} \mathrm{ha}^{-1}$ de glyphosate.

Efeitos antagônicos também são observados na avaliação com 60 DAT (Figura 4) para as três doses de glyphosate. O tratamento com associação de glyphosate e triazinas na dose de $900 \mathrm{~g} \mathrm{ha}^{-1}$ de glyphosate não diferiu do glyphosate aplicado sozinho na dose de $540 \mathrm{~g} \mathrm{ha}^{-1}$.

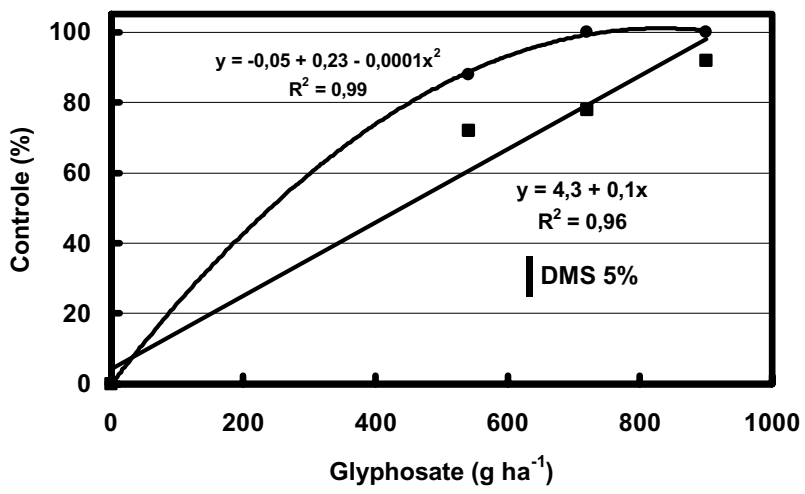

Figura 3 - Controle de sorgo avaliado aos 45 dias após a aplicação de glyphosate isoladamente $(\bullet)$ ou associado a triazinas (ם). Barra vertical para comparar médias de tratamentos entre equações pelo teste $\mathrm{t}(\mathrm{P}<0,05)$. Todas as equações foram significativas $(\mathrm{P}<0,05)$. Eldorado do Sul-RS, 2002.

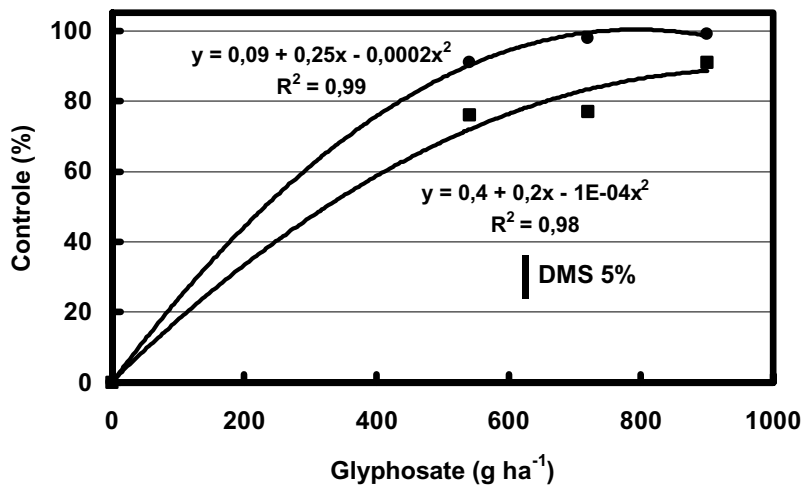

Figura 4 - Controle de sorgo avaliado aos 60 dias após a aplicação de glyphosate isoladamente $(\bullet)$ ou associado a triazinas $(\boldsymbol{\square})$. Barra vertical para comparar médias de tratamentos entre equações pelo teste $t(\mathrm{P}<0,05)$. Todas as equações foram significativas $(\mathrm{P}<0,05)$. Eldorado do Sul-RS, 2002. 


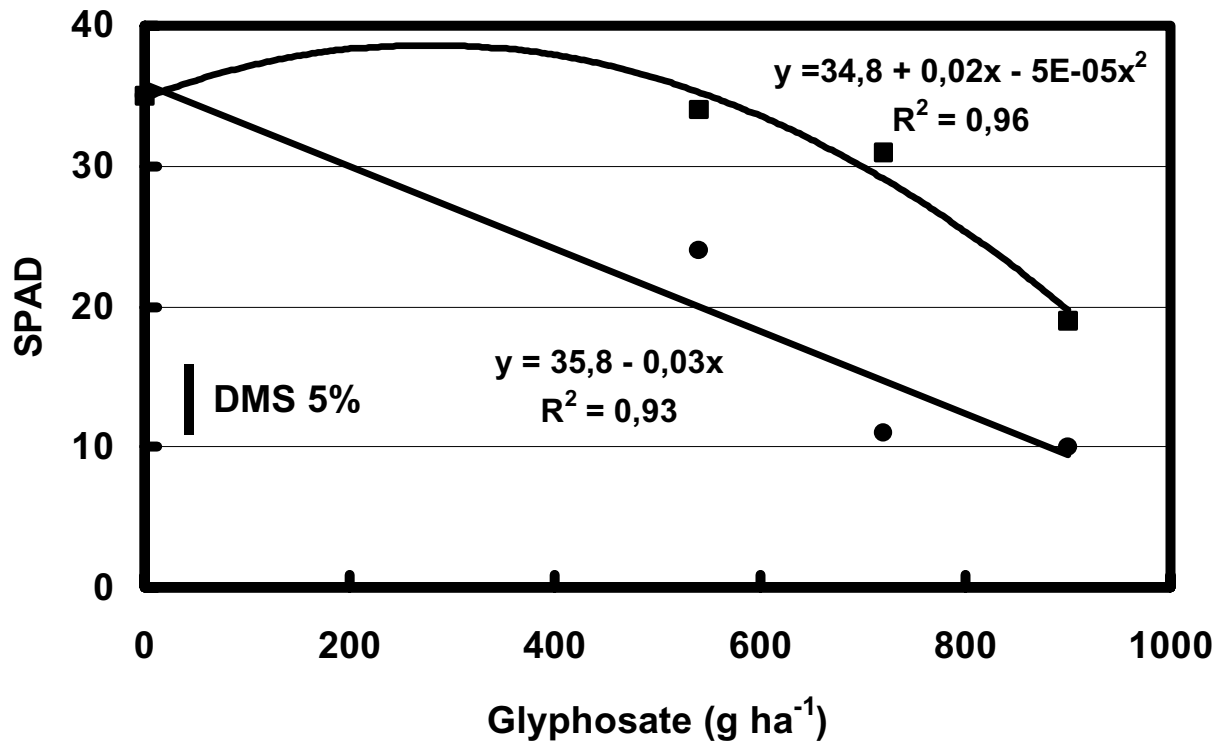

Figura 5 - Leitura de SPAD em plantas de sorgo realizada aos 30 dias após a aplicação de glyphosate isoladamente $(\bullet)$ ou associado a triazinas (ם). Barra vertical para comparar médias de tratamentos entre equações pelo teste $\mathrm{t}(\mathrm{P}<0,05)$. Todas as equações foram significativas $(\mathrm{P}<0,05)$. Eldorado do Sul-RS, 2002.

Os resultados de controle de sorgo confirmam a hipótese de que as triazinas antagonizam a ação de glyphosate. Trabalhos realizados por Appleby \& Somabhi (1978) e Selleck \& Baird (1981) sugerem que ocorra adsorção de glyphosate pelos compostos coloidais contidos na formulação das triazinas. Comparando os tratamentos de glyphosate aplicados sozinho ou em associação com as triazinas, constatou-se que na dose de $900 \mathrm{~g} \mathrm{ha}^{-1}$ houve maior controle de sorgo do que nas demais doses testadas. Isso sugere que, quando utilizado na maior dose, mais moléculas de glyphosate devem ter ficado disponiveis na solução de aspersão, reduzindo o antagonismo das triazinas testadas.

Analisando os resultados de controle do sorgo, verifica-se que os efeitos antagônicos são mais acentuados nas primeiras avaliações, diminuindo com o decorrer do tempo (Figuras 1 a 4). A redução dos efeitos de antagonismo com o decorrer do tempo também foi observada em associações de glyphosate com triazinas e amidas (Selleck \& Baird, 1981).

Os efeitos antagônicos observados nas avaliações de controle do sorgo também podem ser comprovados pela análise do teor de clorofila (SPAD) (Figura 5): observa-se maior teor de clorofila para os tratamentos de glyphosate associado às triazinas, quando comparado ao glyphosate isoladamente.

Os resultados confirmam a hipótese de que a associação de glyphosate com triazinas provoca efeitos antagônicos, reduzindo a eficiência do glyphosate no controle de sorgo. O antagonismo é mais intenso logo após a aplicação, diminuindo com o decorrer do tempo ou com o aumento da dose do glyphosate.

\section{LITERATURA CITADA}

APPLEBY, A.P.; SOMABHI, M. Antagonistic effect of atrazine and simazine on glyphosate activity. Weed Sci., v. 26, n. 2, p. 135-139, 1978.

BORGES. G. O. Resumo histórico do plantio direto no Brasil. In: EMBRAPA-CNPT, FUNDACEP-FECOTRIGO, FUNDAÇÃO ABC. Plantio direto no Brasil. Passo Fundo: Aldeia Norte, 1993. p. 13-17.

BRADLEY, P. R.; JOHNSON, W. G.; SMEDA, R. J. Response of sorghum (Sorghum bicolor) to atrazine, ammonium sulfate, and glyphosate. Weed Technol., v. 14, n. 1 , p. $15-18,2000$.

Planta Daninha, Viçosa-MG, v.21, n.2, p.301-306, 2003 
DAMALAS, C. A.; ELEFTHEROHORINOS, I. G. Dicamba and atrazine antagonism on sulfonylurea herbicides used for Johnsongrass (Sorghum halepense) control in corn (Zea mays). Weed Technol., v. 15, n. 1, p. 62-67, 2001.

FLECK, N. G.; VIDAL, R. A. Inibidores do fotossistema 2. In: VIDAL, R.A.; METOTTO JR., A. (Eds.)

Herbicidologia. Porto Alegre: Evangraf, 2001. p. 100-112.

KRUSE, N. D.; TREZZI, M. M.; VIDAL, R. A. Herbicidas inibidores da EPSPs: Revisão de literatura. R. Bras. Herb. v. 1, n. 2, p. 139-146, 2000.

SELLECK, G. W.; BAIRD, D. D. Antagonism with glyphosate and residual herbicide combinations. Weed Sci., v. 29, n. 2, p. 185-190, 1981.

SHAW, D. R.; ARNOLD, J. C. Weed control from herbicide combinations with glyphosate. Weed Technol., v. 16, n. 1, p. 1-6, 2002

STAHLMAN, P. W.; PHILLIPS, W. M. Inhibition of glyphosate phytotoxicity. Weed Sci., v. 27, n. 5, p. 575-577, 1979.
STARKE, R. J.; OLIVER, L. R. Interaction of glyphosate with chlorimuron, fomesafen, imazetaphyr, and sulfentrazone. Weed Sci., v. 46, n. 6, p. 652-660, 1998.

TREZZI, M. M.; KRUSE, N. D.; VIDAL, R. A. Inibidores de EPSPs. In: VIDAL, R.A.; METOTTO JR., A. (Eds.) Herbicidologia. Porto Alegre: Evangraf, 2001. p. 37-45.

VANGESSEL, M. J.; AYENI, A. O.; MAJEK, B. A. Optimum glyphosate timing with or without residual herbicides in glyphosate-resistant soybeans (Glycine max) under full-season conventional tillage. Weed Technol. v. 14, n. 1, p. 140-149, 2000.

VELlosO, J. A. R. O.; SOUZA, R. O. Plantas daninhas no sistema de plantio direto. In: EMBRAPA-CNPT, FUNDACEP-FECOTRIGO, FUNDAÇÃO ABC. Plantio direto no Brasil. Passo Fundo: Aldeia Norte, 1993. p. 6175 .

VIDAL, R. A.; SPADER, V. Herbicidograma para Commelina benghalensis. Pesticidas: R. Ecotoxicol. Meio Ambiente, v. 9, p. 95-102, 1999. 